\title{
Editorial
}

\section{Functional Nanomaterials for Sustainable Energy Technologies}

\author{
Jun Chen, ${ }^{1}$ Guang Zhu, ${ }^{1}$ Weiqing Yang, ${ }^{2}$ Jin Yang, ${ }^{3}$ Long Lin, ${ }^{1}$ and Yaqing Bie \\ ${ }^{1}$ School of Materials Science and Engineering, Georgia Institute of Technology, Atlanta, GA 30332-0245, USA \\ ${ }^{2}$ School of Materials Science and Engineering, Southwest Jiaotong University, Chengdu 610031, China \\ ${ }^{3}$ Department of Optoelectronic Engineering, Chongqing University, Chongqing 400044, China \\ ${ }^{4}$ Department of Physics, Massachusetts Institute of Technology, Cambridge, MA 02139, USA
}

Correspondence should be addressed to Jun Chen; jun@gatech.edu

Received 12 April 2016; Accepted 13 April 2016

Copyright ( $\odot 2016$ Jun Chen et al. This is an open access article distributed under the Creative Commons Attribution License, which permits unrestricted use, distribution, and reproduction in any medium, provided the original work is properly cited.

As we are facing increasing challenges of diminishing fossil fuel and global warming in the new century, in the past decades, increasing research efforts have been committed to seek for clean and renewable energy sources as well as develop renewable energy technologies. Searching for renewable energy with reduced carbon emissions is mandatory for the sustainable development human civilization. Functional nanomaterials are advanced nanotechnology materials with internal or external dimensions on the order of nanometers. These extremely small dimensions make them unique in energy harvesting, conversion, and storage.

This special issue contributed original research articles as well as review articles that will stimulate the continuing efforts to explore the functional materials for energy applications, especially to develop new types of nanomaterials based energy technologies for clean and renewable energy harvesting/conversion/storage.

Relying on the coupling between triboelectrification and electrostatic induction, the newly developed triboelectric nanogenerators (TENGs) have been proven to be a costeffective, simple, and robust approach for ambient mechanical energy harvesting. Z. Lin et al. presented a comprehensive review of the recent progress in triboelectric nanogenerator as a renewable and sustainable power source. Four working modes of TENGs, vertical contact-separation mode, inplane sliding mode, single-electrode mode, and free-standing triboelectric-layer mode, are, respectively, introduced. And the capability of TENGs for harvesting various kinds of mechanical energies such as vibration, rotation, wind, human motion, and even large-scale water wave energy is systematically demonstrated. This review article will facilitate the advancement of the field of triboelectric nanogenerators for ambient mechanical energy harvesting.

S.-H. Cha also delivered a comprehensive review to this special issue on the recent development of nanocomposite membranes for vanadium redox flow batteries. They have received considerable attentions owning to their long cycle life, flexible design, fast response time, deep-discharge capability, and low pollution emissions in large-scale energy storage. This review article systematically summarized the recent progress in developing nanocomposite membranes with reduced vanadium ion permeability and improved proton conductivity in order to achieve high performance and long life of vanadium redox flow battery systems.

Quantum dot (QD) sensitized hybrid solar cells were also discussed in this special issue. A. Jamshidi et al. presented a colloidal synthesis of a novel type II Mn-doped ZnSe/CdS core/shell QD system as sensitizer with different Mn concentration $(0-3 \%)$ and test its utilization in quantum dot sensitized solar cells. To the best of their knowledge, this was the first time that Mn-doped $\mathrm{ZnSe} / \mathrm{CdS}$ core/shell QDs are successfully synthesized by the method of hot injection and used as a strategy to boost solar cell efficiency. The experimental results suggest that band structure manipulation in type II core/shell nanostructure offers an effective way to improve light harvesting and control of charge transfer via efficient charge separation in sensitized solar cells and that Mn-doping opens a new window to increase device efficiency.

$\mathrm{X}$. Ji et al. also contributed another story about dyesensitized solar cell (DSSC) to this special issue. In the work,

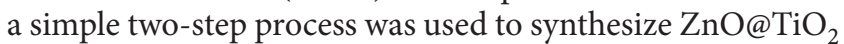
core-shell nanorod thin films, which was demonstrated to 
be utilized as anode materials for dye-sensitized solar cells. And an enhancement of performance was observed by the core-shell structured DSSCs (the PEC of 4.85\%), compared to the bare $\mathrm{ZnO}$ nanorods DSSCs (3.3\%), which was mainly ascribed to a combination of the increasing of loading dye, reduced recombination, and high electron mobility.

In order to enhance the stability of direct methanol fuel cell (DMFC), J. Liu et al. designed a double-layered catalyst cathode electrode containing $\mathrm{Pt} / \mathrm{CeO}_{2}-\mathrm{C}$ as inner catalyst layer and $\mathrm{Pt} / \mathrm{C}$ as outer catalyst layer, which arises from the free-radical scavenger ability of $\mathrm{CeO}_{2}$ in fuel cell without effecting proton conductivity of polymer electrolyte membrane and the convenient preparation. The experimental results show that the membrane electrode assembly with double-layered catalyst cathode possesses high durability in comparison with the conventional one.

The high piezoelectricity and high quality factor ferroelectric thin films are important for electromechanical applications, especially the microelectromechanical system. T. Zhang et al. gave a systematical study of the $0.06 \mathrm{PMnN}$ 0.94PZT (45/55) thin films and nondoped PZT (45/55) thin films on silicon substrates, which were deposited by magnetron sputtering method. And they took a further step to compare the thin film properties with each other as well as the same composition thin films on $\mathrm{MgO}$ substrates. And the crystal structures, piezoelectricity, ferroelectricity, and the dielectricity were also extensively studied, which will be greatly beneficial to future development of thin film based self-power MEMS system.

$\mathrm{H}$. Zhu et al. systematically investigated the miscibility, stability, intermolecular interaction, and nanostructure of monolayer of DPPG/HD binary mixed system in twodimensional states by using the method of LB and AFM technology. The results show that the two components in the binary system are miscible and formed nonideal monolayers at the air/water interface from the analysis of surface pressure-area isotherms.

The thin film bulk acoustic resonators (FBARs) are extensively applied for filters, resonators, and sensors. In the paper of T. Zhang et al., the input acoustic impedance equation with transfer matric method was theoretically derived. And the effect of thickness and density of electrode on the resonance frequency of FBAR was investigated. In addition, the effects of thickness, density, and acoustic velocity on the effective electromechanical coupling factor were, respectively, studied. The presented results in this paper indicate that the thickness, density, and acoustic velocity of electrode are very important, and they can be applied to optimize and design different kind of FBARs.

The manuscripts in this issue are involved in diverse topics but share a transversal common point: functional nanomaterials for sustainable energy technologies. We sincerely hope that the works presented in this special issue will render the readers useful information and facilitate the further development of the research field. 

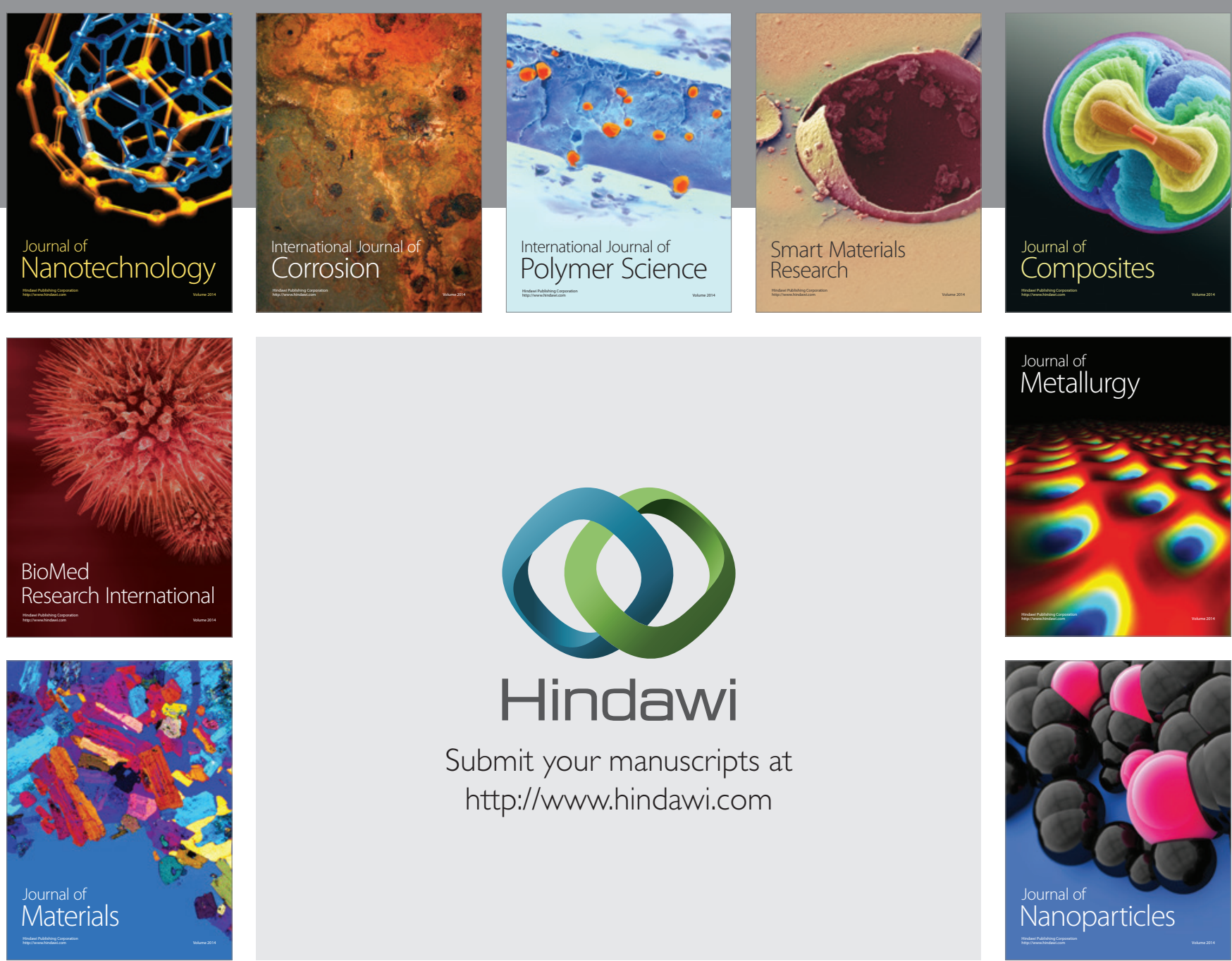

\section{Hindawi}

Submit your manuscripts at

http://www.hindawi.com

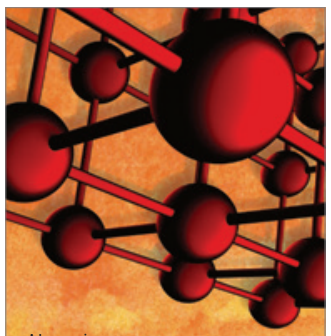

Materials Science and Engineering
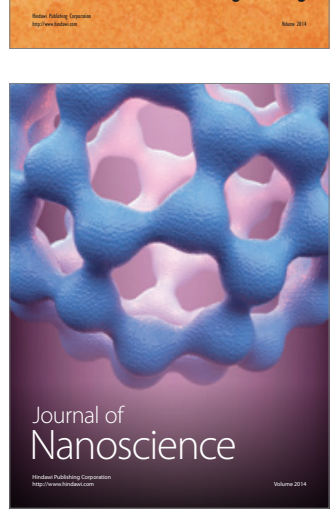
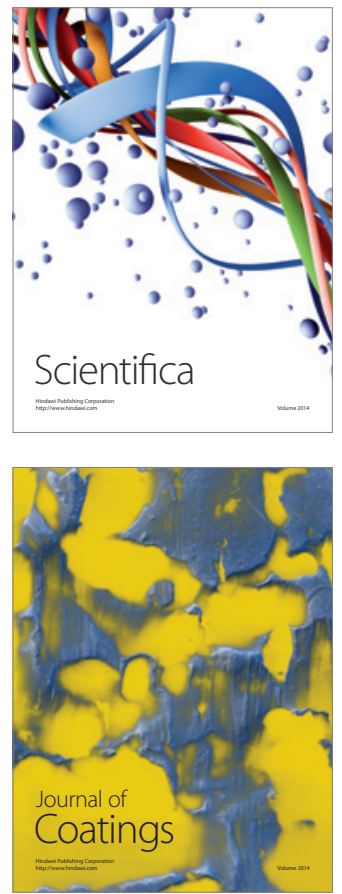
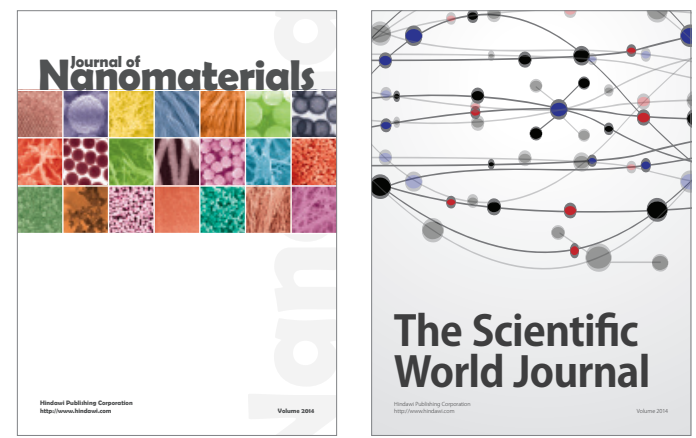

The Scientific World Journal
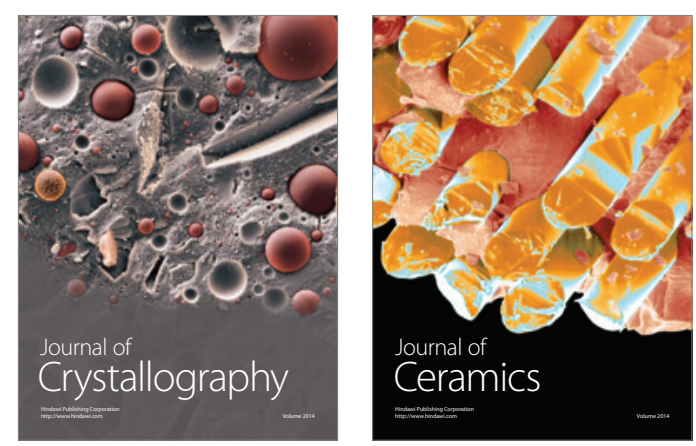
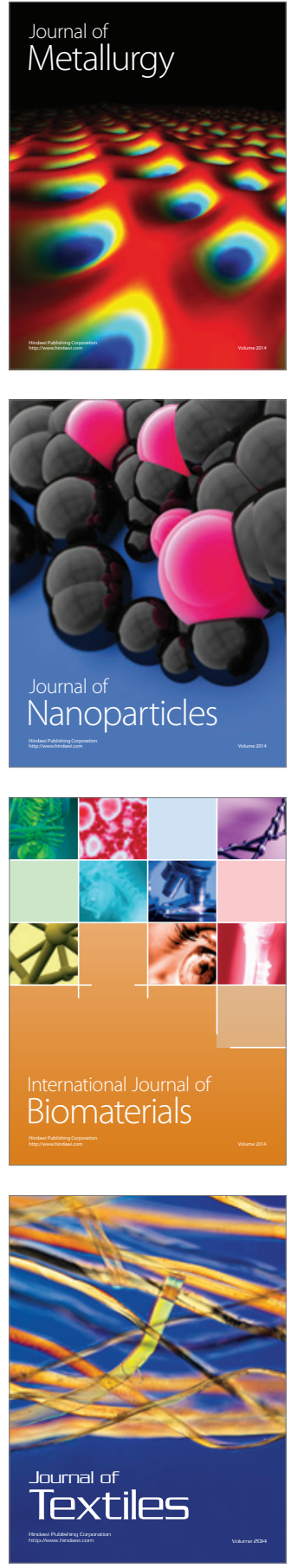